* Dept. of Orthopedics and Traumatology, Hong Hui Hospital ,Xi'an Jiaotong UniversityHealth science center Shaanxi, China

** Dept. of Orthopaedic Surgery University of California San Francisco, USA

Background There are multiple reports on venous thromboembolism (VTE) for major orthopaedic surgery. The incidence and risk factors of VTE in tibial plateau fractures reported less commonly. This study aimed to investigate the incidence and risk factors of clinically important venous thromboembolism(CIVTE) in patients with tibial plateau fractures.

Methods A total of 462 patients aged 18 years and older were retrospectively identified as undergoing operative fixation of tibial plateau fractures from 2003 to 2018 . All the suspected CIVTE patients were examined by the ultrasonography or/and CT scan. Univariate and multivariate analysis were used to assess the association in patient's demographics between CIVTE and no VTE. Variables that were significant at $P$ $<0.05$ in univariate analyses entered into a multivariable logistic regression model to evaluate the risk factors.

Results Thirty-nine (8.4\%) of 462 patients developed CIVTE in $75.5 \%(394 / 462)$ of patients with the chemical prophylaxis. Pulmonary embolism (PE) and deep vein thrombosis DVT was examined in $18(3.9 \%)$ and $21(4.54 \%)$ patients respectively.

\section{Table1 Incidence of CIVTE}

\begin{tabular}{|c|c|c|c|c|c|}
\hline & & & & \multirow[b]{2}{*}{$0.000^{*}$} \\
\hline Variable & N (\%) & $\begin{array}{l}\text { Extremity } \\
\text { injury }\end{array}$ & $\begin{array}{l}104(2 \\
4.6 \%) \\
\end{array}$ & $\begin{array}{l}23(59 . \\
0 \%)\end{array}$ & \\
\hline VTE & $39(8.44 \%)$ & & & & \\
\hline DVT & $21(4.55 \%)$ & Comorbidities & & & \\
\hline $\begin{array}{l}\text { Upper } \\
\text { extremity DVT }\end{array}$ & $8(1.73 \%)$ & Cancer & $\begin{array}{l}8(1.9 \\
\%) \\
\end{array}$ & $\begin{array}{l}5(12.8 \\
\%) \\
\end{array}$ & 0.001 \\
\hline $\begin{array}{l}\text { Lower } \\
\text { extremity DVT* }\end{array}$ & $13(2.81 \%)$ & $\begin{array}{l}\text { Fixation } \\
\text { methods }\end{array}$ & & & 0.000 \\
\hline $\begin{array}{l}\text { PE } \\
\text { DVT with PE }\end{array}$ & $\begin{array}{c}18(3.90 \%) \\
2(0.43 \%)\end{array}$ & One-staged & \begin{tabular}{|l|}
$287(67$ \\
$.8 \%)$ \\
\end{tabular} & $\begin{array}{l}14(35 . \\
9 \%)\end{array}$ & \\
\hline & & Two-staged & $\begin{array}{l}136(32 \\
.2 \%) \\
\end{array}$ & $\begin{array}{l}25(64 . \\
1 \%)\end{array}$ & \\
\hline
\end{tabular}

Table 2 Demography of patients with

\section{CIVTE and no VTE}

\begin{tabular}{|c|c|c|c|c|c|c|c|c|c|}
\hline \multirow{2}{*}{ variable } & \multirow{2}{*}{$\begin{array}{l}\text { No } \\
\text { VTE }\end{array}$} & \multirow{2}{*}{ CIVTE } & \multirow{2}{*}{ total } & \multirow{2}{*}{$\begin{array}{l}\mathrm{P} \\
\text { value }\end{array}$} & Touiqunet & & & & 0.042 \\
\hline & & & & & Yes & $\begin{array}{l}239(5 \\
6.5 \%) \\
\end{array}$ & $\begin{array}{l}15(38 . \\
5 \%)\end{array}$ & & \\
\hline \multicolumn{5}{|l|}{ Gender } & \multirow{2}{*}{ No } & $184(4$ & $24(61$. & & \\
\hline \multirow[t]{2}{*}{ Male } & \multirow{2}{*}{$\begin{array}{l}274 \\
(64.8 \\
\%)\end{array}$} & \multirow{2}{*}{$\begin{array}{l}35 \\
(89.7 \\
\%)\end{array}$} & \multirow{2}{*}{$\begin{array}{l}309( \\
66.9 \\
\%)\end{array}$} & \multirow[t]{2}{*}{0.02} & & $3.5 \%)$ & $5 \%)$ & & \\
\hline & & & & & ICU & & & & 0.000 \\
\hline Open & & & & 0.021 & YES & $\begin{array}{l}34(8.0 \\
\%)\end{array}$ & $\begin{array}{l}24(38 . \\
5 \%)\end{array}$ & & \\
\hline $\begin{array}{l}\text { tracture } \\
\text { Yes }\end{array}$ & $34(8.0$ & $8(20.5$ & & & No & $\begin{array}{l}389(9 \\
2 \%)\end{array}$ & $\begin{array}{l}15(61 . \\
5 \%)\end{array}$ & & \\
\hline no & \begin{tabular}{|l|}
$389(9)$ \\
$2 \%)$ \\
\end{tabular} & $\begin{array}{l}\% 1(79 . \\
5 \%) \\
\end{array}$ & & & $\begin{array}{ll}\text { Injury } & \text { to } \\
\text { definite } & \\
\text { oneration } & \end{array}$ & $\begin{array}{l}7.9 \pm 7 . \\
6\end{array}$ & $\begin{array}{l}13.7 \pm 1 \\
0.6\end{array}$ & $\begin{array}{l}8.38 \\
\pm 8.0 \\
7\end{array}$ & 0.000 \\
\hline Classification & & & & 0.002 & Operation to & $8.5+1$ & $21.7 \pm 2$ & 9.65 & \\
\hline$|-I I|$ & $\begin{array}{l}237(5 \\
60 \%)\end{array}$ & $\begin{array}{l}12(30 . \\
8 \%)\end{array}$ & & & discharge & 2.5 & 2.5 & $\begin{array}{l} \pm 14 . \\
06\end{array}$ & 0.001 \\
\hline IV-VI & $\begin{array}{l}0.0 \%) \\
186(4 \\
2.3 \%) \\
\end{array}$ & $\begin{array}{l}27(69 . \\
2 \%) \\
\end{array}$ & & & $\begin{array}{l}\text { Length stay } \\
\text { in ICU }\end{array}$ & $\begin{array}{l}0.9 \pm 4 \\
0\end{array}$ & $\begin{array}{l}10.3 \pm 1 \\
4.2\end{array}$ & $\begin{array}{l}14.3 \\
3 \pm 11 \\
54\end{array}$ & 0.000 \\
\hline Associated & & & & & \begin{tabular}{|l|} 
Prophylaxis \\
\end{tabular} & & & & \\
\hline $\begin{array}{l}\text { Injury (mult- } \\
\text { injury) }\end{array}$ & & & & & Yes & $\begin{array}{l}313(7 \\
4 \%)\end{array}$ & $\begin{array}{l}36(92 . \\
3 \%)\end{array}$ & & 0.010 \\
\hline $\begin{array}{l}\text { Traumatic } \\
\text { brain Injury }\end{array}$ & $\begin{array}{l}13(3.1 \\
\%)\end{array}$ & $\begin{array}{l}11(28 . \\
2 \%)\end{array}$ & & $0.000^{*}$ & No & $\begin{array}{l}110(2 \\
6.0 \%)\end{array}$ & $3(7.7 \%$ & & \\
\hline
\end{tabular}

Tips:All the $p>0.05$ were not inclued in the table Table 3. VTE prophylaxis and therapeutic methods

\begin{tabular}{|l|l|l|l|}
\hline $\begin{array}{l}\text { medication } \\
\text { with/without } \\
\text { IVF }\end{array}$ & $\begin{array}{l}\text { prophylaxi } \\
\text { s }\end{array}$ & treatment \\
\hline Enoxaparin & $\begin{array}{l}40 \mathrm{mg}- \\
60 \mathrm{mg} \text { daily }\end{array}$ & $\begin{array}{l}80-120 \mathrm{mg} \\
\text { daily }\end{array}$ \\
\hline Heparin & NA & $\begin{array}{l}5000 \mathrm{U} \\
\text { bid-5000 } \\
\text { tid }\end{array}$ \\
\hline Warfarin & NA & $\begin{array}{l}\text { Depends } \\
\text { on the INR }\end{array}$ \\
\hline Aspirin & $\begin{array}{l}375 \mathrm{mg} \\
\text { daily }\end{array}$ & NA \\
\hline $\begin{array}{l}\text { Enoxaparin/VC } \\
\text { F }\end{array}$ & $\begin{array}{l}40 \mathrm{mg}- \\
60 \mathrm{mg} \\
\text { daily/IVF }\end{array}$ & $\begin{array}{l}80-120 \mathrm{mg} \\
\text { daily/IVF }\end{array}$ \\
\hline
\end{tabular}

Table 4 risk factors of CIVTE in tibial plateau fractures

\begin{tabular}{|c|c|c|c|c|c|c|c|}
\hline & $\beta$ & $\begin{array}{l}\text { S. } \\
E\end{array}$ & $\begin{array}{l}\text { Wal } \\
\mathrm{s}\end{array}$ & $p$ & $\begin{array}{l}\text { Exp } \\
(\beta)\end{array}$ & \multicolumn{2}{|c|}{$95 \% \mathrm{Cl}$} \\
\hline $\begin{array}{l}\text { Gender(male: } \\
\text { female) }\end{array}$ & $\begin{array}{l}2.4 \\
22\end{array}$ & $\begin{array}{l}.77 \\
7\end{array}$ & $\begin{array}{l}9.7 \\
23\end{array}$ & $\begin{array}{l}.0 \\
02\end{array}$ & $\begin{array}{l}11 . \\
267\end{array}$ & $\begin{array}{l}2.4 \\
59\end{array}$ & $\begin{array}{l}51 . \\
63 \\
7\end{array}$ \\
\hline $\begin{array}{l}\text { Traumatic } \\
\text { brain injury }\end{array}$ & $\begin{array}{l}1.5 \\
64\end{array}$ & $\begin{array}{l}.76 \\
4\end{array}$ & $\begin{array}{l}4.1 \\
89\end{array}$ & $\begin{array}{l}.0 \\
41\end{array}$ & $\begin{array}{l}4.7 \\
77\end{array}$ & $\begin{array}{l}1.0 \\
69\end{array}$ & $\begin{array}{l}21 . \\
35 \\
9\end{array}$ \\
\hline $\begin{array}{l}\text { Associated } \\
\text { spine injury }\end{array}$ & $\begin{array}{l}2.2 \\
52\end{array}$ & $\begin{array}{l}.54 \\
6\end{array}$ & $\begin{array}{l}17 . \\
027\end{array}$ & $\begin{array}{l}.0 \\
00\end{array}$ & $\begin{array}{l}9.5 \\
06\end{array}$ & $\begin{array}{l}3.2 \\
62\end{array}$ & $\begin{array}{l}27 \\
70 \\
3\end{array}$ \\
\hline $\begin{array}{l}\text { Associated } \\
\text { extremity } \\
\text { injury }\end{array}$ & $\begin{array}{l}1.3 \\
07\end{array}$ & $\begin{array}{l}.44 \\
1\end{array}$ & $\begin{array}{l}8.7 \\
89\end{array}$ & $\begin{array}{l}.0 \\
03\end{array}$ & $\begin{array}{l}3.6 \\
95\end{array}$ & $\begin{array}{l}1.5 \\
57\end{array}$ & $\begin{array}{l}8.7 \\
69\end{array}$ \\
\hline $\begin{array}{l}\text { Length stay in } \\
\text { ICU }\end{array}$ & $\begin{array}{l}.09 \\
6 \\
\end{array}$ & $\begin{array}{l}.02 \\
9 \\
\end{array}$ & $\begin{array}{l}11 . \\
167\end{array}$ & $\begin{array}{l}.0 \\
01 \\
\end{array}$ & $\begin{array}{l}1.1 \\
01\end{array}$ & $\begin{array}{l}1.0 \\
40 \\
\end{array}$ & $\begin{array}{l}1.1 \\
64 \\
\end{array}$ \\
\hline $\begin{array}{l}\text { From injury to } \\
\text { definite } \\
\text { operation }\end{array}$ & $\begin{array}{l}.06 \\
0\end{array}$ & $\begin{array}{l}.02 \\
1\end{array}$ & $\begin{array}{l}8.1 \\
63\end{array}$ & $\begin{array}{l}.0 \\
04\end{array}$ & $\begin{array}{l}1.0 \\
62\end{array}$ & $\begin{array}{l}1.0 \\
19\end{array}$ & $\begin{array}{l}1.1 \\
06\end{array}$ \\
\hline
\end{tabular}

Conclusions The incidence of CIVTE in tibial plateau fracture was high (8.4\%); The males, associated with traumatic brain injury, associated with spine injury, associated with extremity injury, the time from injury to definite operation, length stayed in ICU were the independent risk factors.

\section{Limitations}

Our study has several limitations. First, the main limitation of the current study is its retrospective nature. Some predictive variables may not have sufficient statistical power, or some important variables may have been ignored. Second, 462 patients during a 15-year period study, such a long period with relatively small sample size may weaken the power to study the incidence of CIVTE. Additionally, the period of the study was relatively long, the guideline of the VTE prophylaxis has changed in several times, which might influence the strategy of VTE prophylaxis and treatment. 